\title{
Cortical dynamics of lateral inhibition: Visual persistence and ISI
}

\author{
GREGORY FRANCIS \\ Purdue University, West Lafayette, Indiana
}

\begin{abstract}
Psychophysical studies show that increasing the interstimulus interval (ISI) between two stimuli decreases persistence of the first stimulus. While some researchers account for these results with interactions of transient and sustained inhibition, this paper describes an alternative explanation. In a neural-network model of boundary detection called the boundary contour system, persistence is the result of feedback-generated reverberations. Mechanisms to control these reverberations include lateral inhibition, which computer simulations show allows persistence in the network to qualitatively match the psychophysical data. Additional simulations predict that increasing the duration of the second stimulus should cause persistence to increase with ISI. The model links psychophysical data on visual persistence with computational requirements of spatial vision and properties of cells in visual cortex.
\end{abstract}

Burr (1980) observed that moving stimuli were not as blurred as would be expected. Studies of visual persistence show that stimuli persist for hundreds of milliseconds (e.g., Bowen, Pola, \& Matin, 1974), which would produce substantial blurring of moving stimuli. However, Burr suggested that mechanisms responsible for detecting motion inhibited persisting representations of a stimulus to deblur the moving percept. Di Lollo and Hogben (1987) found an inverse proximity effect, where nearby stimuli that were spatially close and temporally contiguous reduced the persistence of nonmoving stimuli. This finding suggested that motion-detection mechanisms were not critical for deblurring moving stimuli, and that lateral inhibition interacting across space and time inhibited persisting representations of past stimuli. Farrell, Pavel, and Sperling (1990) showed similar results using stimuli that moved in apparent motion. They also developed a descriptive model of visual persistence that outlined how lateral inhibition from a new stimulus affects the decay of old stimulus representations. In this model, persistence is the result of a fading trace, and lateral inhibition decreases the strength of the trace so that it more quickly decays below a constant threshold.

While the idea of persistence as a fading trace is intuitively appealing, the mechanisms underlying the trace and their interaction with mechanisms of lateral inhibition appear to be more complicated than the Farrell et al. (1990) description. Di Lollo and Hogben (1987) found that the effects of nearby stimuli were stronger with longer stimulus onset asynchronies (SOAs), and Castet (1994), using a method very similar to Farrell et al.'s, demonstrated that persistence of a leading stimulus decreases as

Correspondence should be addressed to the author at Department of Psychological Sciences, 1364 Psychological Science Building, Purdue University, West Layfayette, IN 47907-1364 (e-mail: gfrancis@psych. purdue.edu). the interstimulus interval (ISI) between it and a trailing stimulus increases. Figure 1a shows data for 2 subjects from Castet. Farrell et al.'s model did not consider the possibility that ISI might affect the persistence of the leading stimulus, so without modification it cannot account for these results. In general, the effect of ISI on persistence is seemingly paradoxical for fading trace theories, because increasing the ISI between stimuli should result in less net inhibition and therefore should allow the trace to persist longer. The psychophysical data demonstrate exactly the opposite result.

Other theories emphasize the influence of lateral inhibition rather than the properties of a fading trace. Di Lollo and Hogben (1987) and Castet (1994) suggested that the theory of transient on sustained inhibition (Breitmeyer \& Ganz, 1976) explained the inverse relationship between persistence and ISI (or SOA). In this theory, visual input stimulates two channels: (1) a transient channel with a short response lag and a brief response and (2) a sustained channel with a longer response lag and a long response. In the theory, the duration of persistence matches the duration of the sustained channel, and activity in the transient channel inhibits activity in the sustained channel through lateral inhibition. With the difference in lags, the transient response to the second flash after a short ISI precedes the sustained response to the first flash, so that the sustained response remains relatively uninhibited. As the ISI increases, the inhibitory transient response of the second flash overlaps more fully with the sustained response of the first flash, thereby decreasing persistence of the sustained response and the visual percept. In this theory, persistence is dependent less on a fading perceptual trace and more on the strength of lateral inhibition from transient cells and the overlap of transient and sustained responses.

Francis, Grossberg, and Mingolla (1994) took a different approach by showing that the mechanisms of a neural network theory of visual perception account for some properties of visual persistence. They showed that 
(a)

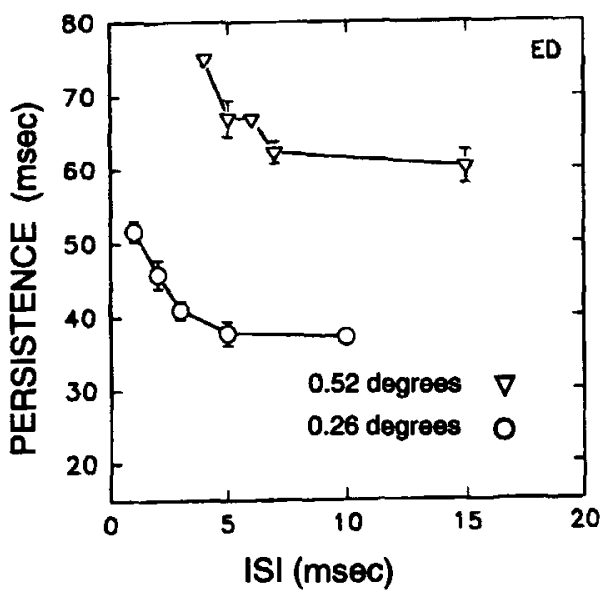

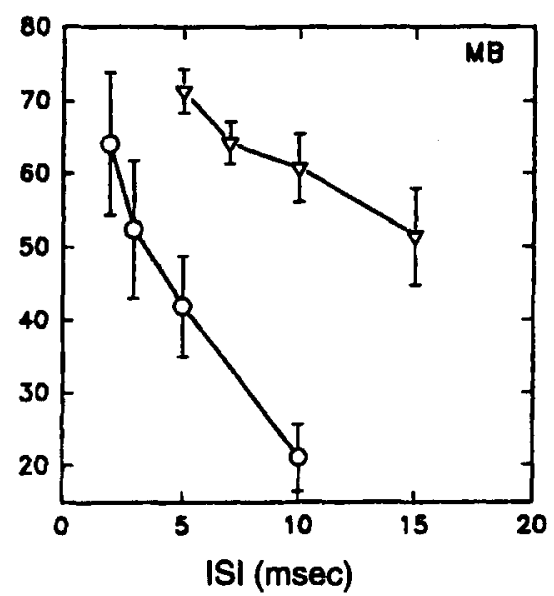

(b)

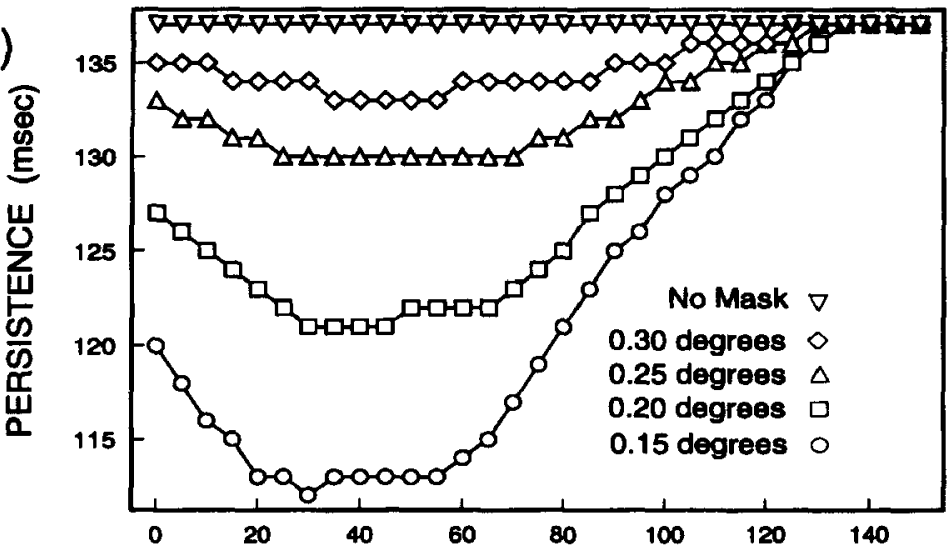

ISI (msec)

Figure 1. Visual persistence as a function of ISI and spatial separation between stimuli. (a) Psychophysical data for 2 subjects. As the ISI increases and as the spatial separation between stimuli decreases, persistence decreases. Two other subjects showed qualitatively similar results. (From "Effect of the ISI on the Visible Persistence of a Stimulus in Apparent Motion," by E. Castet, 1994, Vision Research, 34, pp. 2103-2114. Copyright 1994 by Elsevier Science Ltd. Reprinted with permission.) Results of computer simulations. The simulations show the same trends as the psychophysical data.

excitatory feedback in a neural network model of boundary detection produces hysteresis that accounts for a variety of persistence data. The network does not explicitly model a fading trace, but something like a trace emerges through the network's dynamics. The current paper describes how the model, called the boundary contour system (BCS; Grossberg \& Mingolla, 1985a, 1985b), accounts for Castet's (1994) data. Earlier simulations of the model (Francis et al., 1994) replicated the influence of spatial masking, as well as other properties of visual persistence, but did not investigate the role of ISI between successive stimuli. The next section describes the dynamic properties of the BCS model and summarizes how it explains many properties of visual persistence. Subsequent sections analyze the role of lateral inhibition in the model and demonstrate that the model accounts for the ISI data.

\section{Resonance as a Source of Visual Persistence}

Grossberg and Mingolla (1985a, 1985b) introduced the BCS to model how the visual system detects, completes, and regularizes patterns of oriented edges from retinal images. Such patterns arise from changes in regions of luminance, texture, shading, or stereo signals. BCS computations consist of a series of filtering, competitive, and cooperative stages as schematized in Figure 2 and reviewed in several reports (e.g., Grossberg, 1994). Briefly, pairs of like-oriented simple cells that are sensitive to opposite contrast polarities send their rectified output signals to like-oriented complex cells. Complex cells activate hypercomplex cells through on-center off-surround connections (first competitive stage), whose off-surround carries out an end-stopping operation (Hubel \& Wiesel, 1965; Orban, Kato, \& Bishop, 1979a, 1979b). These interactions are gated by habituative chemical transmitters, 


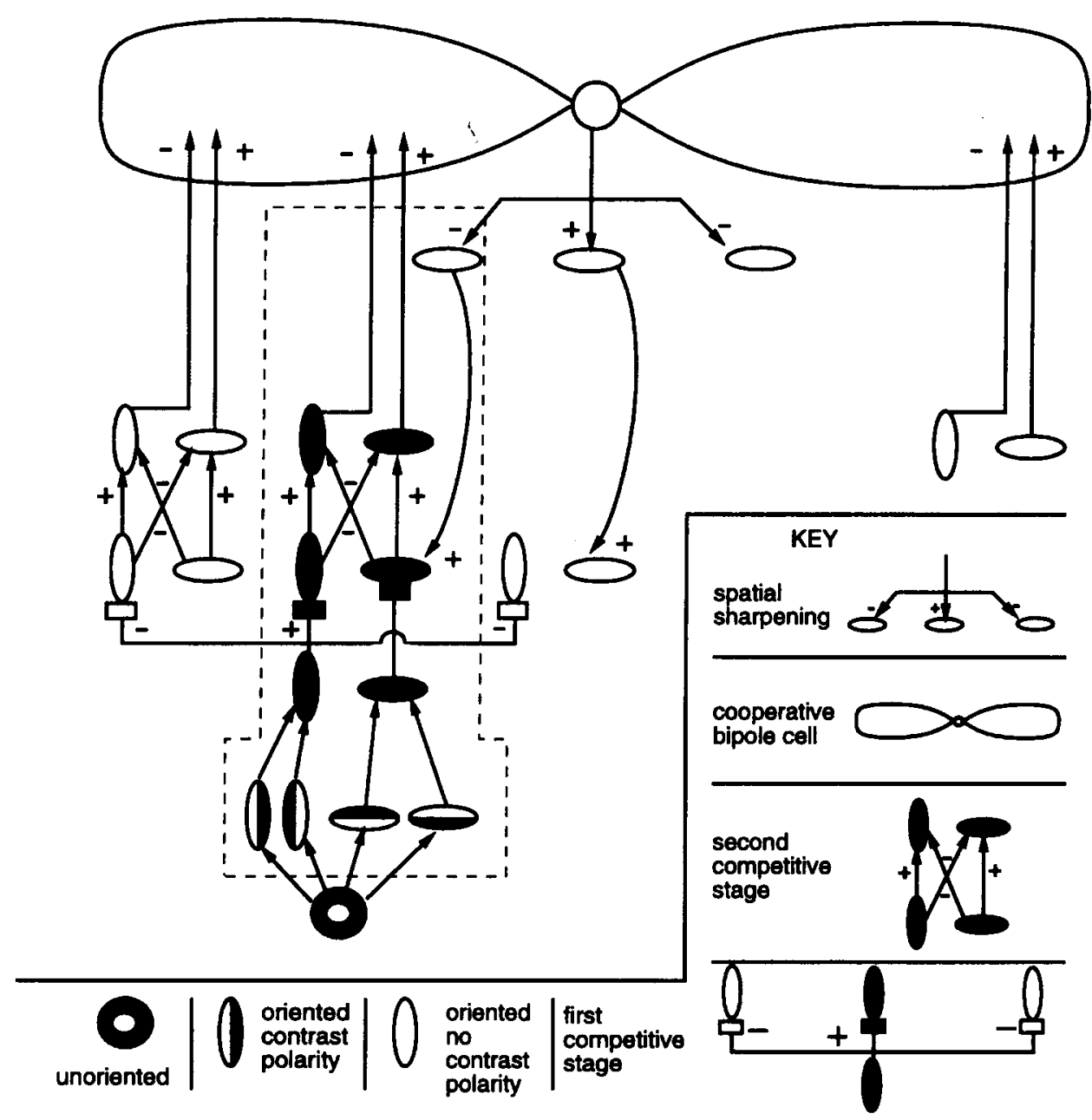

Figure 2. Schematic diagram of the boundary contour system. Cooperative and competitive interactions embedded in a feedback network process visual segmentations.

which pass neural signals across synapses. The hypercomplex cells input to a competition across orientation (second competitive stage) among higher order hypercomplex cells. Their outputs identify the location and orientation of stimulus edges or boundaries. They feed into cooperative bipole cells which, in turn, generate feedback signals that enhance spatially and orientationally consistent oriented patterns of activation, while inhibiting (spatial sharpening) inconsistent ones. Like the cells reported to exist in area V2 (von der Heydt, Peterhans, \& Baumgartner, 1984), a model bipole cell requires substantial excitatory input on each half of its bow-tie-shaped receptive field. Positive feedback among BCS bipole cells and hypercomplex cells helps to drive a cooperative-competitive decision process that completes the statistically most favored patterns and suppresses less favored patterns of activation. Cooperating activations are said to resonate in the network.

Each cell in the BCS has its own local dynamics involving activation by inputs and passive decay (of the order of simulated milliseconds). However, the excita- tory feedback loops dominate the temporal aspects of the $\mathrm{BCS}$. When inputs feed into the BCS, they trigger reverberatory circuits that are not easily stopped. Indeed, simulations in Francis et al. (1994) demonstrate that, if left unchecked, these reverberations can last for hundreds of simulated milliseconds. The reverberations do not last indefinitely, because the spatial structure of the bipole cells' receptive fields limits the persistence of resonating activities. A bipole cell requires excitatory inputs on both sides of its receptive field; thus, when the visual inputs disappear, cells centered on the middle of an image contour receive strong bipole feedback, but cells centered near the end receive no feedback. At stimulus offset, the activity of a cell centered at the end of an image contour passively decays away. This exposes a new cell at the contour end, which stops receiving bipole feedback and passively decays away as well. This erosion process continues from the contour ends to the middle of the contour, as described in Francis et al. (1994). Moreover, as more boundary signals drop out of the feedback loop, the loop contains less activity, thereby further weakening the 
feedback signals. As a result, erosion speeds up over time, until finally the feedback loop no longer contains enough activity to support itself and the resonance collapses.

Erosion occurs slowly relative to the duration of a brief stimulus and, if unchecked, could lead to undesirably long boundary persistence after stimulus offset and thus image smearing in response to image motion. Image smearing does occur for very fast-moving stimuli in some conditions (e.g., Chen, Bedell, \& Öğmen, 1995), and the smear generally produces a trail that narrows to a point, which is what would be expected if visual signals eroded from contour ends toward the middle. However, such smearing is not typically observed, so the problem for the $\mathrm{BCS}$ is to accelerate boundary erosion in response to rapidly changing imagery. More generally, the BCS needs to use resonant feedback to complete patterns of activation for unmoving scenic objects, even as it actively resets the same activations when the objects change to control smearing in a form-sensitive way. Remarkably, the same BCS mechanisms that create resonant boundaries also reset them. Francis et al. (1994) identified two mechanisms embedded in the BCS design that reset resonating patterns. One mechanism, called a gated dipole, produces reset signals at stimulus offset that actively inhibit the persisting activations. Habituation of chemical transmitters (boxes in Figure 2) shifts the balance of activity among competing pathways in the second competitive stage. This shift creates rebounds of activity in nonstimulated pathways at the offset of a stimulus. Within the $\mathrm{BCS}$, these rebounds inhibit the persisting activations. The properties of these reset signals explain why persistence of static stimuli varies inversely with stimulus duration and luminance (e.g., Bowen et al., 1974), why the persistence of illusory contours is greater, and differently affected by stimulus duration, than luminance contours (Meyer \& Ming, 1988), and how orientation-specific adaptation can increase or decrease persistence (Meyer, Lawson, \& Cohen, 1975). Details of these properties are in Francis et al. (1994); for the present analysis, a second mechanism, lateral inhibition, is more significant.

\section{Lateral Inhibition: Simulations}

Lateral inhibition exists in two stages of the BCS architecture: the first competitive stage and spatial sharpening (see Figure 2). In the current simulations, the role of spatial sharpening is insignificant, although including its interactions would not adversely affect the results.

Figure 3a shows the creation and erosion of one row of activations in the feedback loop produced by a brief horizontal bar in a visual display with no subsequent stimulus. This figure shows the temporal response of cells along a cross section of the image plane that follows the contour of the target. Brighter regions indicate higher cell activities, and the contour lines mark curves of equal activity. At stimulus onset (far left), the cell activities rapidly grow in strength, and grow stronger as the excitatory bipole feedback develops. After stimulus offset ( 50 simulated milliseconds, the brightest part of the graph), the activations drop in strength, due to the lack of feed-
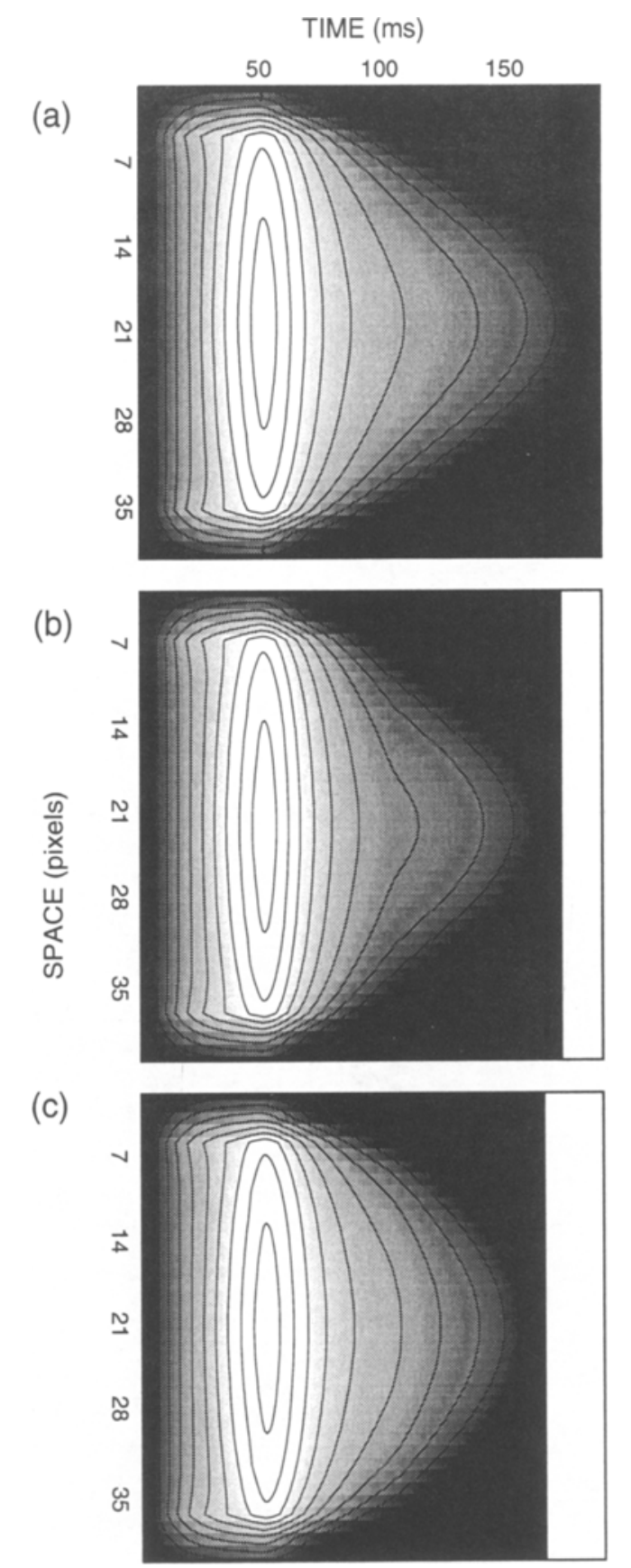

Figure 3. Activation of cells responding to the first stimulus along a cross section of the image plane. Brighter regions indicate stronger boundary-cell activity. The contours mark out equalvalue curves. Offset of all boundary signals occurs at the time indicated at the end of the black background. (a) With no masking stimulus. (b) With a masking stimulus immediately following offset of the first stimulus. (c) With a masking stimulus and an ISI of 50 simulated milliseconds.

forward input from the visual image, and the activations erode from the contour ends toward the middle, as described above. The speeding up of erosion is evidenced by the inward bend to the outer contour line, as a constant rate of erosion would produce a straight line. The end of 
the graph indicates disappearance of all boundary signals for the stimulus. To calculate persistence $(136 \mathrm{msec})$, subtract the duration the stimulus was physically present $(50 \mathrm{msec})$ from the time all signals disappear $(186 \mathrm{msec})$.

Figure $3 b$ shows the erosion of the same contour when the onset of a second stimulus ( 0.15 simulated visual degrees away) immediately follows the offset of the first. The activities of cells responding to the second stimulus are not visible in Figure $3 \mathrm{~b}$ because such cells lie on a different cross section of the image plane, but through the first competitive stage their inhibitory signals weaken the resonating feedback loop responsible for the persistence of the activities generated by the first stimulus. As a result, the erosion of activities progresses more rapidly than in the single stimulus case (Figure 3a), so that all activations disappear by $169 \mathrm{msec}$ from stimulus onset. However, because the inhibition of the first competitive stage depends on the visual input and not on the activities in the feedback loop, it is short-lived, lasting only slightly longer than the duration of the second stimulus ( 50 simulated milliseconds). The change in direction of the contour lines at around $100 \mathrm{msec}$ indicates that the rate of erosion has slowed down because the inhibition has weakened. Thus, the persisting signals of the first stimulus outlast the inhibition generated by the second stimulus. In general, though, the inhibition causes the model cell activities to erode more quickly, as a comparison of Figures $3 \mathrm{a}$ and $3 \mathrm{~b}$ shows.

Figure $3 \mathrm{c}$ shows the erosion of the first stimulus contour when the second stimulus follows an ISI of $50 \mathrm{sim}-$ ulated milliseconds. During the 50 -msec ISI, the activities generated by the first stimulus weaken and erode, so when the inhibition from the second stimulus arrives, it has a much greater effect on the remaining activities. This inhibition causes the remaining activities to erode so quickly that they disappear sooner (162 msec from stim- ulus onset) than they would if the inhibition had appeared after a short ISI (Figure 3b).

This nonlinear effect of lateral inhibition is due to the nature of persistence in the feedback loop. In both the 0 and $50-\mathrm{msec}$ ISI simulations, the inhibitory signals sent from the second stimulus to the first are the same. Activities in the feedback loop are less sensitive to that inhibition for a short ISI because the resonance in the loop is strong. After the longer ISI, the resonance has weakened and the remaining activities are more sensitive to the inhibition. As a result, increasing the ISI over a limited range, while causing the inhibition to arrive later, decreases the persistence of signals. Figure $1 \mathrm{~b}$ shows simulation results measuring the persistence of first stimulus signals as a function of ISI and spatial separation from the second stimulus. The results are qualitatively similar to the data over the range of ISIs studied by Castet (1994), shown in Figure 1a.

\section{Predictions}

As the ISI grows very long, activities generated by the first stimulus almost completely erode before the inhibition from the second stimulus arrives. Thus, further increases in the ISI allow persisting activities to remain uninhibited for greater lengths of time. Most models would predict similar results, since, if the ISI is long enough, the second stimulus can hardly affect the first. Figure $1 \mathrm{~b}$ shows these trends.

A second prediction of the model concerns the role of stimulus duration. If the second stimulus has a longer stimulus duration, then the lateral inhibition of the first competitive stage can last throughout the erosion of the first stimulus activations. This long-term inhibition produces results opposite those reported by Castet (1994), who used stimuli of very short durations. Figure 4 plots

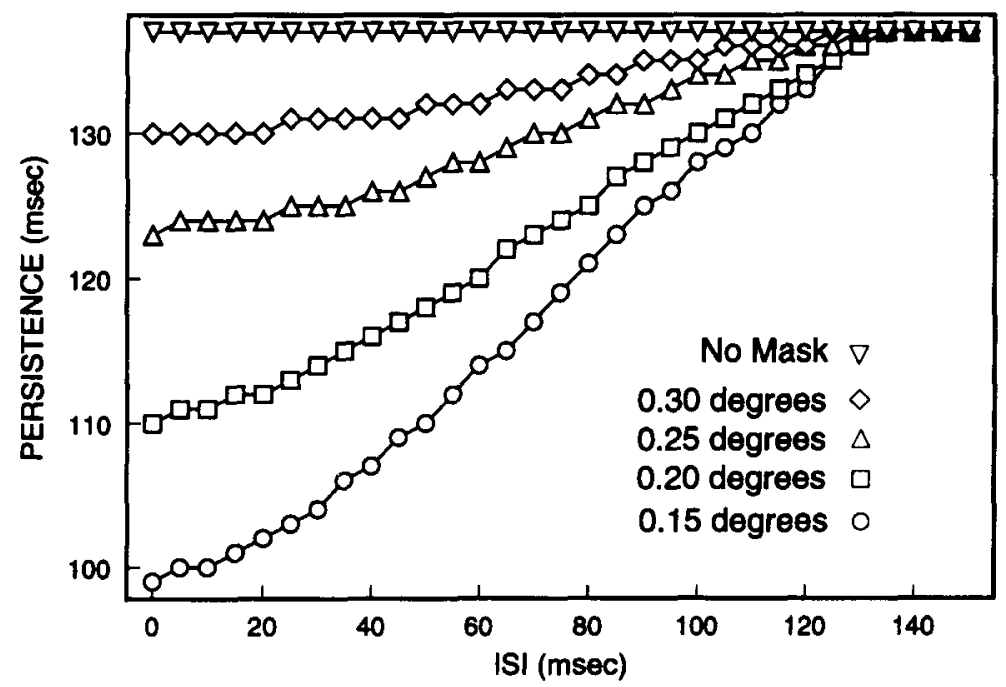

Figure 4. Boundary persistence as a function of ISI and spatial separation when the second stimulus has a duration of 300 simulated milliseconds. The longer duration of lateral inhibition continues throughout the persistence of the first stimulus boundary erosion. As a result, persistence increases with ISI. This result is a testable prediction of the role of the first competitive stage. 
the results of simulations when the second stimulus duration is 300 simulated milliseconds. Unlike the results in Figure $1 \mathrm{~b}$, increasing the ISI for this display always results in longer persistence, because the first stimulus receives less inhibition. Results by Groner, Bischof, and Di Lollo (1989) and Dixon and Di Lollo (1994) appear to be consistent with this prediction. They showed that performance on a form-completion task worsened as the duration of the second half of the form (second stimulus) increased. This prediction is also consistent with related studies of metacontrast masking, such as Breitmeyer's (1978) demonstration that the strength of metacontrast masking increases with the duration of the masking stimulus.

The model also makes predictions concerning neurophysiological characteristics. The BCS model suggests that the persistence of bipole cortical analogues reported by von der Heydt et al. (1984) should be qualitatively similar to the psychophysical data. It should also be possible to observe the inward erosion of activations by sampling from a single bipole cell in visual cortex. After finding the center of the bipole cell's receptive field, one could run a series of experiments varying the position of luminance edges relative to the center of the cell's receptive field. Because the model predicts that signals erode from the contour ends, the cell should show its greatest persisting response when its receptive field is centered on the contour and should show less persistence as the experimenter shifts the contour center to one side or the other of the receptive-field center. These results should hold when the luminance edge extends beyond the cell's spatial receptive field, and should follow despite the fact that the model predicts that cell activations would not vary by position when the stimulus was physically present.

The lateral inhibition responsible for the decrease in persistence is hypothesized to correlate with the inhibition involved in creating end-stopped hypercomplex cells. End-stopped cells studied by Orban et al. (1979b) had an average spatial range of $1.9^{\circ}$, which is large enough to account for the lateral inhibition in Castet (1994), Di Lollo and Hogben (1987), and Farrell et al. (1990). Additional research suggests that end-stopped cells are built from combinations of non-end-stopped cells. Bolz and Gilbert (1986), for example, show that reversibly inactivating Layer 6 in the cat reduces or abolishes end-stopping in the superficial layers. These properties are consistent with the first competitive stage in the BCS architecture. Moreover, the BCS's explanation of the persistence data suggests that inactivation of Layer 6 should destroy the masking effects of adjacent stimuli on judged persistence. If similar effects can be found in monkey cortex, the inactivation of endstopping inhibition should also produce slower erosion of bipole cell activities in a masking display.

\section{Discussion}

The goal of this study was to identify neural network mechanisms that robustly explain the properties of vi- sual persistence. As in Francis et al. (1994), the properties described here hold across a wide range of parameter choices. The relationship between persistence and ISI is due to the basic network design, rather than to any particular parameter choices. Such robustness is highly desirable, because it suggests that even as the BCS model evolves to fit other properties of visual perception, it will continue to explain the persistence data. Indeed, to make the current simulations computationally feasible, the receptive fields of cells in the BCS model were "stripped down" relative to simulations that study the spatial characteristics of the BCS. Because of these simplifications, the simulations do not yet allow the model to produce quantitative fits to persistence data or to generate quantitative predictions about persistence.

The BCS model has some similarities with and some differences from the transient-sustained theories, which Castet (1994) and Di Lollo and Hogben (1987) suggested could account for the effect of ISI on persistence. The theories are similar in that they both suggest that lateral inhibition accounts for the effect of spatial separation on persistence. In both models, the fall off of lateral inhibition with distance accounts for the effect of separation on persistence duration. On the other hand, they propose quite different mechanisms to explain the effect of ISI. In the BCS model, the inhibitory signals sent from the second stimulus to the first are essentially the same regardless of the ISI. The effect of those signals on persistence depends on the strength of the resonating activations of the first stimulus. After a longer ISI, the resonating signals are weaker and the inhibition has a bigger impact on the remaining signals. In the transient--sustained theory, activations from the first stimulus have a smaller role. The strength of transient inhibition varies with ISI due to the relative lags between transient and sustained channels, so that, as ISI increases, the masking stimulus generates stronger overlapping inhibition with the first stimulus and shorter persistence. Thus, these two theories offer quite different explanations of the data on ISI and persistence. To adequately compare these models, proponents of the transient-sustained theory should move beyond descriptive explanations and build a computational model of the theory to test its ability to account for the data.

Significantly, the BCS is part of a computational model of visual perception and not a model of visual persistence per se. Persistence of signals in the BCS emerges from dynamic interactions that are necessary to process spatial characteristics of visual inputs. That other studies use the same model mechanisms to explain characteristics of texture segregation, shape-from-shading, brightness perception, and three-dimensional vision provides strong evidence in support of those mechanisms. Thus, the model architecture links data that appear to be distinct by considering psychophysical, neurophysiological, and computational properties of visual processing. 


\section{REFERENCES}

Bolz, J., \& Gilbert, C. (1986). Generation of end-inhibition in the visual cortex via interlaminar connections. Nature, 320, 362-365.

Bowen, R., Pola, J., \& Matin, L. (1974), Visual persistence: Effects of flash luminance, duration and energy. Vision Research, 14, 295-303.

BREITMEYER, B. (1978). Metacontrast masking as a function of mask energy. Bulletin of the Psychonomic Society, 12, 50-52.

BREITMEYER, B., \& GANZ, L. (1976). Implications of sustained and transient channels for theories of visual pattern masking, saccadic suppression, and information processing. Psychological Review, 83, 1-36.

BURR, D. (1980). Motion smear. Nature, 284, 164-165.

CASTET, E. (1994). Effect of the ISI on the visible persistence of a stimulus in apparent motion. Vision Research, 34, 2103-2114.

Chen, S., Bedell, H., \& ÖĞMeN, H. (1995). A target in real motion appears blurred in the absence of other proximal moving targets. Vision Research, 35, 2315-2328.

Di Lollo, V., \& Hogben, J. (1987). Suppression of visible persistence as a function of spatial separation between inducing stimuli. Perception \& Psychophysics, 41, 345-354.

Dixon, P., \& Di Lollo, V. (1994). Beyond visible persistence: An alternative account of temporal integration and segregation in visual processing. Cognitive Psychology, 26, 33-63.

Farrell, J., Pavel, M., \& SPerling, G. (1990). The visible persistence of stimuli in stroboscopic motion. Vision Research, 30, 921-936.

Francis, G., Grossberg, S., \& Mingolla, E. (1994). Cortical dynamics of feature binding and reset: Control of visual persistence. Vision Research, 34, 1089-1104.

Groner, M., Bischof, W., \& Di Lollo, V. (1989). A model of visible persistence and temporal integration. Spatial Vision, 3, 293-304.

GrossberG, S. (1994). 3-D vision and figure-ground separation by visual cortex. Perception \& Psychophysics, 55, 48-120.

Grossberg, S., \& Mingolla, E. (1985a). Neural dynamics of form perception: Boundary completion, illusory figures, and neon color spreading. Psychological Review, 92, 173-211.

Grossberg, S., \& Mingolla, E. (1985b). Neural dynamics of perceptual grouping: Textures, boundaries, and emergent segmentations. Perception \& Psychophysics, 38, 141-171.

Hubel, D., \& Wiesel, T. (1965). Receptive fields and functional architecture in two nonstriate visual areas (18 and 19) of the cat. Journal of Neurophysiology, 28, 229-289.

LUDTKE, D. (1992). NXPlot3d (Version 3.0) [Computer software]. Houston, TX: Rice University, Department of Physics. Available WWW: http://ion.rice.edu/nsci/plotting/nxplot3d.html

MEYER, G., LAwSON, R., \& COHEN, W. (1975). The effects of orientationspecific adaptation on the duration of short-term visual storage. $V i$ sion Research, 15, 569-572.

Meyer, G., \& Ming, C. (1988). The visible persistence of illusory contours. Canadian Journal of Psychology, 42, 479-488.

Orban, G., Kato, H., \& Bishop, P. (1979a). Dimensions and properties of end-zone inhibitory areas in receptive fields of hypercomplex cells in cat striate cortex. Journal of Neurophysiology, 42, 833-849.

Orban, G., KaTO, H., \& Bishop, P. (1979b). End-zone region in receptive fields of hyper-complex and other striate neurons in the cat. Journal of Neurophysiology, 42, 818-832.

von der Heydt, R., Peterhans, E., \& Baumgartner, G. (1984). Illusory contours and cortical neuron responses. Science, 224, 1260-1262.

\section{APPENDIX Simulation Details}

The network equations are identical to those used in Francis et al. (1994). Readers interested in the equations are referred to that paper.

The following parameters were used: $A=67.5, B=2.5$, $C=60.0, D=0.05, H=0.1, J=20.0, K=0.0003, L=3.0$, $M=5.0, N=25.0, P=0.0005, Q=0.5, R=0.61, T=0.3$, $V=10.0, \alpha=0.5, \beta=3.0, \gamma=1.5, \delta=3.0$. With the exception of parameters $N$ and $V$, these are the same values used in Francis et al. (1994). These parameter modifications do not change the analysis of the BCS reported in Francis et al. (1994), although some of the quantitative results may vary. (Note that parameter $V$ was a constant in Equation A19 of Francis et al. (1994). In the original study, its value of 1 was given in the equation rather than being properly listed as a parameter.)

All computations were on a $15 \times 40$ pixel grid. As in Francis et al. (1994), symmetry arguments required computation of only one half of the long side of the simulation, thereby reducing the computation to a $15 \times 20$ grid. The first stimulus for each simulation consisted of a luminance bar (2.0 simulated footlamberts) 1 pixel wide and 30 pixels long on a dark background $(0.000001$ simulated footlamberts). The second stimulus consisted of a bar of equal luminance and size located 3 to 6 pixel spaces from the first ( 1 pixel space corresponds to 0.05 visual degrees). Further separation was beyond the range of lateral inhibition in the model and had no effect on the persistence of the first stimulus. Except for the simulations used to produce Figure 4 , where the second stimulus lasted 30 time units ( 300 simulated milliseconds), both the first and second stimuli lasted five time units (corresponding to 50 simulated milliseconds). The ISI between the stimuli varied from 0 simulated milliseconds to 150 simulated milliseconds in steps of 5 .

Persistence for the simulations in Figure $1 \mathrm{~b}$ was measured by noting the time beyond stimulus offset when all the Level 6 signals corresponding to the first stimulus dropped below a constant threshold value of 0.5 units. To save computation, the BCS simulations included only horizontal cells (the orientation of the stimuli). All equations were solved using Euler's method with a step size of 0.01 . Activities of cells were checked every 0.1 time steps ( 1 simulated millisecond) to measure persistence. Each curve in Figure $l b$ took approximately 1 day to calculate on a dedicated IBM RS6000 355 POWERstation. The density plots in Figure 3 were made with the program NXPlot3d (Ludtke, 1992).

(Manuscript received July 17,1995 ; revision accepted for publication January 14, 1996.) 